\title{
Homotopy Meaningful Hybrid Model Structures
}

\author{
Aaron D. Ames
}

\begin{abstract}
Hybrid systems are systems that display both discrete and continuous behavior and, therefore, have the ability to model a wide range of robotic systems such as those undergoing impacts. The main observation of this paper is that systems of this form relate in a natural manner to very special diagrams over a category, termed hybrid objects. Using the theory of model categories, which provides a method for "doing homotopy theory" on general categories satisfying certain axioms, we are able to understand the homotopy theoretic properties of such hybrid objects in terms of their "non-hybrid" counterparts. Specifically, given a model category, we obtain a "homotopy meaningful" model structure on the category of hybrid objects over this category with the same discrete structure, i.e., a model structure that relates to the original non-hybrid model structure by means of homotopy colimits, which necessarily exist. This paper, therefore, lays the groundwork for "hybrid homotopy theory."
\end{abstract}

\section{Introduction}

Hybrid systems are systems that display both continuous and discrete behavior and so have important applications to robotic systems, e.g., mechanical systems undergoing impacts such as bipedal robotic walkers are naturally modeled by systems of this form. As with dynamical systems, understanding the homotopy-theoretic properties of hybrid systems-including topology and homology-allows for important insights into the behavior of these systems. Unlike dynamical systems, there is currently no such mathematical framework. The goal of this paper is to provide the first steps toward establishing such a framework.

Fundamental to our investigations is the theory of model categories, which provides a method for "doing homotopy theory" on general categories with three distinguishable classes of morphisms (weak equivalences, fibrations and cofibrations) which satisfy certain axioms. Originally formulated by Quillen in [Qui67], model category theory has since blossomed into a full-fledged area of research capable of addressing homotopy-theoretic questions in a general context. Some of the quintessential model categories are the category of topological spaces, the category of simplicial sets and the category of chain complexes - the model structure of these

2000 Mathematics Subject Classification. Primary 18G55, 18A25; Secondary 18D99, 18A30. Key words and phrases. Hybrid systems, model categories, homotopy colimits.

This research was supported in part by NSF award \#CCR-0225610. 
categories plays a fundamental role in algebraic topology and homology. Therefore, understanding hybrid systems in the context of model categories will allow one to understand the homotopy-theoretic properties of these systems, laying the groundwork for hybrid homotopy theory.

The core observation of this work is that hybrid systems, and more generally hybrid objects, can be represented equivalently as diagrams over a category. That is, given a category $M$ consisting of the non-hybrid objects of interest, e.g., topological spaces, a hybrid object over this category consists of a small category $\mathcal{D}$ of a very specific form, termed a $D$-category, that captures the discrete structure of the hybrid object together with a functor:

$$
\mathbf{A}: \mathcal{D} \rightarrow \mathrm{M},
$$

that captures the continuous structure of the hybrid object. Therefore, given a category $M$, we are interested in studying the functor category $M^{\mathcal{D}}$.

This paper explores the theory of model categories in the light of hybrid objects. For a $\mathrm{D}$-category $\mathcal{D}$, this amounts to finding a homotopy meaningful model structure on $M^{\mathcal{D}}$ given a model structure on $M$; that is, the goal is a model category structure that yields homotopy colimits - the total left derived functor of colim. More specifically, it is desirable to find a model category structure on $\mathrm{M}^{\mathcal{D}}$ in which:

For every weak equivalence $\vec{f}: \mathbf{A} \rightarrow \mathbf{B}$ between cofibrant objects

$\mathbf{A}$ and $\mathbf{B}$ in $\mathbf{M}^{\mathcal{D}}, \operatorname{colim}(\vec{f})$ is a weak equivalence.

The model structure on $\mathrm{M}^{\mathcal{D}}$ thus is said to be cofibrantly homotopy meaningful. For such a model structure, the colimit induces a functor

$$
\text { hocolim : } \mathrm{Ho}\left(\mathrm{M}^{\mathcal{D}}\right) \rightarrow \mathrm{Ho}(\mathrm{M})
$$

between homotopy categories, termed the homotopy colimit, which is given by

$$
\operatorname{hocolim}(\mathbf{A}) \cong \operatorname{colim}\left(\mathbf{A}^{\prime}\right)
$$

with $\mathbf{A}^{\prime}$ any cofibrant object weakly equivalent to $\mathbf{A}$.

The main result of this paper is: Given a model category $\mathrm{M}$, there is a cofibrantly homotopy meaningful model structure on $\mathrm{M}^{\mathcal{D}}$. Therefore, homotopy colimits exist and relate the model structure of $M^{\mathcal{D}}$ to that of $M$; that is, there is a direct relationship between "hybrid homotopy theory" and the "non-hybrid homotopy theory" from which it is derived. The connection between hybrid objects and hybrid systems implies that we thus have derived a homotopy theory of hybrid systems with the same discrete structure.

\section{Hybrid Systems}

As the central topic of this paper is abstract, it is important to devote some effort to establishing its relationship to robotic systems, i.e., the goal of this section is to justify all subsequent constructions. We begin by introducing the definition of a general hybrid system. In order to better understand how hybrid systems naturally arise in the context of robotic systems, we then will discuss hybrid Lagrangians and the associated hybrid systems; further details can be found in [Ame06a] and [AS06].

Definition 2.1. A hybrid system is a tuple

$$
\mathfrak{H}=(\Gamma, D, G, R, X),
$$


where

- $\Gamma=(Q, E)$ is an oriented graph (possibly infinite), i.e., $Q$ and $E$ are sets and there exist source and target functions, sor : $E \rightarrow Q$ and tar : $E \rightarrow Q$; the source and target of edge $e \in E$ is thus given by sor $(e)$ and $\operatorname{tar}(e)$, respectively.

- $D=\left\{D_{i}\right\}_{i \in Q}$ is a set of domains, where $D_{i}$ is a smooth manifold.

- $G=\left\{G_{e}\right\}_{e \in E}$ is a set of guards, where $G_{e} \subseteq D_{\text {sor }(e)}$ is an embedded submanifold of $D_{\text {sor }(e)}$.

- $R=\left\{R_{e}\right\}_{e \in E}$ is a set of reset maps; these are smooth maps $R_{e}: G_{e} \rightarrow$ $D_{\operatorname{tar}(e)}$.

- $X=\left\{X_{i}\right\}_{i \in Q}$ is a collection of vector fields, i.e., $X_{i}: D_{i} \rightarrow T D_{i}$ is a vector field on the manifold $D_{i}$.

Roughly speaking, a hybrid system has both a "discrete" and a "continuous" component. The discrete component is the graph $\Gamma$, and the continuous component is the collection of data $(D, G, R, X)$. That is, one can roughly view a hybrid system as a collection of dynamical systems $\left(D_{i}, X_{i}\right), i \in Q$, with $D_{i}$ a manifold and $X_{i}$ a vector field on that manifold, interacting based upon the guard and reset maps in a way that is consistent with the discrete structure, $\Gamma$.

2.1. Trajectories of Hybrid Systems. As with dynamical systems, one can consider trajectories of a hybrid system. Unlike dynamical systems, these trajectories tend to display an abundance of "pathological" behavior, e.g., Zeno behavior and non-uniqueness of solutions. Since this paper is devoted to the topological properties of hybrid systems, we will not formally introduce trajectories for these systems although we will briefly discuss some of their salient properties in the context of an example; we refer the reader to [Ame06a] for a formal definition.

EXAMPLE 2.2. To demonstrate the way in which non-uniqueness can arise in hybrid systems due to the spacial configuration of the domains, guards and reset maps, we will consider a specific example. The simplicity of this example indicates the prevalence of such behavior.

Consider a hybrid system $\mathfrak{H}=(\Gamma, D, G, R, X)$ with

- $\Gamma=(Q, E)$, where $Q=\{0,1,2\}$ and $E=\left\{e_{1}=(0,1), e_{2}=(0,2)\right\}$.

- $D=\left\{D_{0}, D_{1}, D_{2}\right\}$, where $D_{i}=[0, \infty)$ for $i=1,2,3$.

- $G=\left\{G_{e_{1}}, G_{e_{2}}\right\}$, where $G_{e_{i}}=\{0\}$ for $i=1,2$.

- $R=\left\{R_{e_{1}}, R_{e_{2}}\right\}$, where $R_{e_{1}}(0)=1$ and $R_{e_{2}}(0)=2$.

- $X=\left\{X_{0}, X_{1}, X_{2}\right\}$, where $X_{0}(x)=-1, X_{1}(x)=1$ and $X_{2}(x)=2$.

The motivation for considering this hybrid system is that, as a result of the fact that the guards are not disjoint, it displays non-uniqueness (or nondeterminism or branching of solutions). To demonstrate how this non-uniqueness presents itself, we will explicitly construct trajectories of the system. (Note that the notion of trajectory considered in this example is specifically kept simple to avoid confusion; the formal definition of trajectories is necessarily more involved [Ame06a].)

Let us consider trajectories of $\mathfrak{H}$ over the time interval $[0, \infty)$ with initial conditions in $D_{0}$. In this case, for some $x_{0} \in D_{0}$, the system will evolve according to the solution of the vector field $X_{0}$ until the guard $G_{e_{1}}=G_{e_{2}}$ is reached, i.e., until $c_{0}(\tau)=0$ for some $\tau \geq 0$ with $c_{0}(t)$ the solution to $X_{0}$. At this point, the trajectory can do one of two things: (1) jump to domain $D_{1}$ and evolve according 
to the solution of the vector field $X_{1}$ with initial condition $R_{e_{1}}\left(c_{0}(\tau)\right)$ or $(2)$ jump to domain $D_{2}$ and evolve according to the solution of the vector field $X_{2}$ with initial condition $R_{e_{2}}\left(c_{0}(\tau)\right)$. Therefore, we see that for every initial condition $x_{0} \in D_{0}$ there exist two trajectories ${ }^{1}$ over the time interval $[0, \infty)$ with initial condition $x_{0}$ :

$$
\begin{aligned}
& \chi_{1}(t)=\left\{\begin{array}{ccc}
-t+x_{0} & \text { if } & 0 \leq t \leq x_{0} \\
t-x_{0}+1 & \text { if } & x_{0}<t<\infty
\end{array}\right. \\
& \chi_{2}(t)=\left\{\begin{array}{ccc}
-t+x_{0} & \text { if } & 0 \leq t \leq x_{0} \\
2\left(t-x_{0}\right)+2 & \text { if } & x_{0}<t<\infty
\end{array}\right.
\end{aligned}
$$

since, necessarily, $\tau=x_{0}$. Thus $\mathfrak{H}$ does not have unique solutions.

Example 2.2 illustrates a couple of important points regarding the definition of a hybrid system. Firstly, it demonstrates the role that the guards and reset maps play in relation to one anther-the reset maps dictate how, when a guard is reached, the state of the system changes; this is why, for example, $R_{e}: G_{e} \rightarrow D_{\operatorname{tar}(e)}$ for all $e \in E$. Example 2.2 also illustrates the importance of the spacial interaction between of the guards, domains and reset maps, e.g., how overlapping guards can result in non-uniqueness. These considerations only serve to further motivate the study of the topological properties of hybrid systems, since they are fundamentally topological in nature.

We will now discuss hybrid systems in the context of robotic systems undergoing impacts, i.e., hybrid systems as they relate to hybrid Lagrangians, in order to further motivate the consideration of hybrid systems. First, we briefly recall:

2.2. Lagrangians. Consider a configuration space $Q$ which is assumed to be a smooth manifold. The equations of motion for a mechanical (or robotic) system (cf. [MLS93]) are typically obtained from a Lagrangian $L: T Q \rightarrow \mathbb{R}$ given in coordinates by:

$$
L(q, \dot{q})=\frac{1}{2} \dot{q}^{T} M(q) \dot{q}-V(q)
$$

where $M(q)$ is the inertial matrix, $\frac{1}{2} \dot{q}^{T} M(q) \dot{q}$ is the kinetic energy and $V(q)$ is the potential energy. In this case, the Euler-Lagrange equations yield:

$$
M(q) \ddot{q}+C(q, \dot{q}) \dot{q}+N(q)=0,
$$

where $C(q, \dot{q})$ is the Coriolis matrix and $N(q)=\frac{\partial V}{\partial q}(q)$. Setting $x=(q, \dot{q})$, we obtain the Lagrangian vector field, $X_{L}: T Q \rightarrow T(T Q)$, associated to $L$ :

$$
\dot{x}=X_{L}(x)=\left(\dot{q}, M(q)^{-1}(-C(q, \dot{q}) \dot{q}-N(q))\right) .
$$

We thus have associated to a Lagrangian a dynamical system $\left(T Q, X_{L}\right)$, the behavior of which describes the behavior of the robotic system modeled by $L$.

2.3. Hybrid Lagrangians. In order to enter the realm of hybrid systems, suppose that there is a unilateral constraint function $h: Q \rightarrow \mathbb{R}$, i.e., a function that dictates the admissible configurations of the system, $\{q \in Q: h(q) \geq 0\}$, which usually arise from physical constraints on the system.

\footnotetext{
${ }^{1}$ In general, trajectories of hybrid systems take multiple values at the switching times (in this case $\tau$ ); here we defined trajectories that take single values at this time for the sake of simplicity.
} 

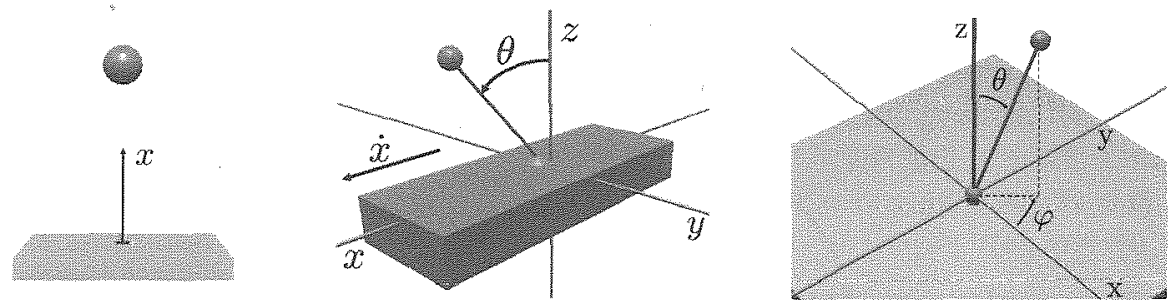

Figure 1. (Left) Bouncing ball. (Center) Pendulum on a cart. (Right) Spherical pendulum mounted on the ground.

Definition 2.3. A hybrid Lagrangian is a tuple:

$$
\mathfrak{L}=(Q, L, h),
$$

where

- $Q$ is a configuration space.

- $L: T Q \rightarrow \mathbb{R}$ is a Lagrangian.

- $h: Q \rightarrow \mathbb{R}$ is a unilateral constraint function such that 0 is a regular value of $h$, i.e., $h^{-1}(0)$ is a smooth manifold.

Systems that are described by hybrid Lagrangians arise naturally, e.g., a ball bouncing on a flat surface where the ball is not allowed to pass through the ground, a pendulum mounted on a cart when the pendulum is not allowed to pass through the cart, and a pendulum mounted on the ground where the pendulum is not allowed to pass through the ground; these examples are illustrated pictorially in Figure 1. In this paper, we will consider the following simple example:

EXAMPLE 2.4. Consider a ball bouncing on the ground in one dimension; see Figure 1 (Left). In this case:

$$
\mathfrak{L}^{\text {ball }}=\left(Q^{\text {ball }}, L^{\text {ball }}, h^{\text {ball }}\right)
$$

where $Q^{\text {ball }}=\mathbb{R}$ with $L^{\text {ball }}(x, \dot{x})=\frac{1}{2} m\|\dot{x}\|^{2}-m g x$. Finally, the constraint that the ball is not allowed to pass through the ground is manifested in the constraint function $h^{\text {ball }}(x)=x$.

2.4. Hybrid systems from hybrid Lagrangians. Just as one can associate to a Lagrangian a dynamical system, one can associated to a hybrid Lagrangian $\mathfrak{L}=(Q, L, h)$ a hybrid system:

$$
\mathfrak{y}^{\mathfrak{L}}=\left(\Gamma^{\mathfrak{L}}, D^{\mathfrak{L}}, G^{\mathfrak{L}}, R^{\mathfrak{L}}, X^{\mathfrak{L}}\right) .
$$

The discrete component of $\mathfrak{H}^{\mathfrak{L}}$ is a graph $\Gamma^{\mathfrak{L}}$ consisting of a single vertex and edge:

$$
\Gamma^{\mathfrak{L}}=\overbrace{b}^{a}
$$

Therefore, $D^{\mathfrak{L}}=\left\{D_{b}^{\mathfrak{L}}\right\}, G^{\mathfrak{L}}=\left\{G_{a}^{\mathfrak{L}}\right\}, R^{\mathfrak{L}}=\left\{R_{a}^{\mathfrak{L}}\right\}$ and $X^{\mathfrak{L}}=\left\{X_{b}^{\mathfrak{L}}\right\}$, i.e., hybrid systems obtained from hybrid Lagrangians consist of a single domain, guard, rest map and vector field. This data is obtained directly from the hybrid Lagrangian $\mathfrak{L}$ in the manner described below. 


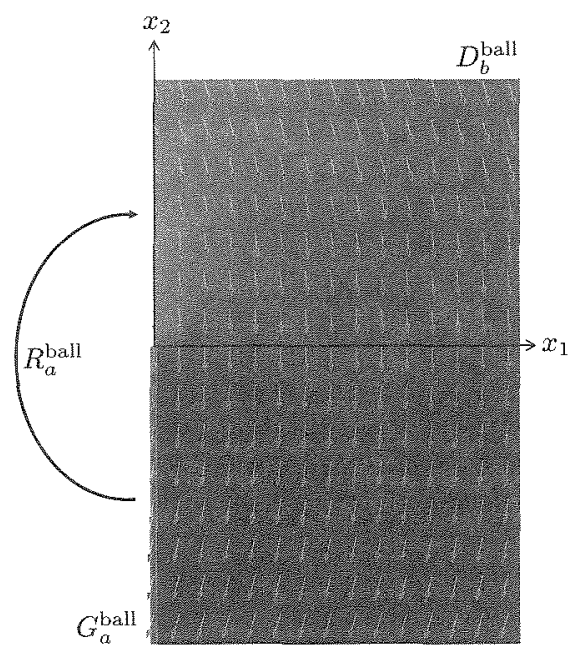

FIGURE 2. The hybrid model of a bouncing ball

From the constraint function $h$ one obtains the domain:

$$
D_{b}^{\mathfrak{L}}=\{(q, \dot{q}) \in T Q: h(q) \geq 0\},
$$

which is the space on which the continuous behavior of the hybrid system evolves according to the vector field $X_{b}^{\mathfrak{L}}:=X_{L}$, the vector field obtained from the Lagrangian $L$. The "hybrid" nature of the system exerts itself when the guard,

$$
G_{a}^{\mathfrak{L}}=\left\{(q, \dot{q}) \in T Q: h(q)=0 \text { and } d h_{q} \dot{q} \leq 0\right\},
$$

is reached, i.e., when a trajectory reaches the guard, an instantaneous transition in the velocity occurs. This change in velocity is dictated by the reset map:

$$
R_{\alpha}^{\mathfrak{L}}(q, \dot{q})=(q, P(q, \dot{q}))
$$

where in coordinates:

$$
P(q, \dot{q})=\dot{q}-(1+e) \frac{d h_{q} \dot{q}}{d h_{q} M(q)^{-1} d h_{q}^{T}} M(q)^{-1} d h_{q}^{T},
$$

which is obtained using the classical Newtonian impact equations [Bro99]. Here $0 \leq e \leq 1$ is the coefficient of restitution, e.g., for a perfectly elastic impact $e=1$, and for a perfectly plastic impact $e=0$.

EXAMPLE 2.5. Continuing with Example 2.4, using the above constructions we obtain a hybrid system modeling a ball bouncing in one dimension:

$$
\mathfrak{5}^{\text {ball }}=\left(\Gamma^{\text {ball }}, D^{\text {ball }}, G^{\text {ball }}, R^{\text {ball }}, X^{\text {ball }}\right),
$$

with $\Gamma^{\text {ball }}$ as in (2.1), from the hybrid Lagrangian $\mathfrak{2}^{\text {ball }}=\left(Q^{\text {ball }}, L^{\text {ball }}, h^{\text {ball }}\right)$.

Let $x_{1}$ and $x_{2}$ denote the position and velocity of the bouncing ball, respectively. The domain and guard for the hybrid system are obtained from $h^{\text {ball }}$ as follows:

$$
\begin{aligned}
& D_{b}^{\text {ball }}=\left\{\left(x_{1}, x_{2}\right) \in \mathbb{R}^{2}: x_{1} \geq 0\right\}, \\
& G_{a}^{\text {ball }}=\left\{\left(x_{1}, x_{2}\right) \in \mathbb{R}^{2}: x_{1}=0, \quad x_{2} \leq 0\right\},
\end{aligned}
$$

which encodes the fact that the position always must be positive and that a transition in the velocities of the system should occur when the position is zero and 
the velocity is "downward pointing." The reset map for the system is given by $R_{a}^{\text {ball }}\left(x_{1}, x_{2}\right)=\left(x_{1},-e x_{2}\right)$, where $0 \leq e \leq 1$ is the coefficient of restitution for the ball; this map encodes the fact that when the ball impacts the ground, its velocity is reversed and scaled down by the amount of energy lost through impact. Finally, the vector field for this system is given by $X_{b}^{\text {ball }}\left(x_{1}, x_{2}\right)=\left(x_{2},-g\right)$, where $g$ is the acceleration due to gravity. A graphical representation of this hybrid system can be seen in Figure 2.

2.5. Hybrid spaces. As with dynamical systems, it is sometimes desirable to consider the underlying "space" of a hybrid system. This amounts to "forgetting" the vector field on each domain along with the smooth structure of the other data defining a hybrid system. More specifically, we introduce the following:

DeFinition 2.6. A (topological) hybrid space is a tuple:

$$
\mathbb{H}=(\Gamma, D, G, R),
$$

where

- $\Gamma=(Q, E)$ is an oriented graph.

- $D=\left\{D_{i}\right\}_{i \in Q}$ where $D_{i}$ is a topological space.

- $G=\left\{G_{e}\right\}_{e \in E}$ where $G_{e}$ is a subspace of $D_{\text {sor }(e)}$.

- $R=\left\{R_{e}\right\}_{e \in E}$ where $R_{e}: G_{e} \rightarrow D_{\operatorname{tar}(e)}$ is a continuous map.

It will be demonstrated in the next section that hybrid spaces correspond to hybrid objects over the category of topological spaces: hybrid topological spaces. We first describe this correspondence in the context of hybrid systems obtained from hybrid Lagrangians.

2.6. Topology and hybrid systems. The goal of this paper is to better understand the topology of hybrid systems and, therefore, we will consider hybrid topological spaces obtained from hybrid systems. To illustrate this construction in the context of mechanical systems, we will show that the "topological" portion of the hybrid system obtained from a hybrid Lagrangian, i.e., the data $\Gamma^{\mathfrak{L}}, D^{\mathfrak{L}}$, $G^{\mathfrak{L}}$ and $R^{\mathfrak{L}}$, can be equivalently represented as a diagram over the category of topological spaces.

Recall that the underlying "discrete" structure of the hybrid system obtained from a hybrid Lagrangian is the graph $\Gamma^{\mathfrak{L}}$ as given in (2.1). This graph can be transformed into a small category $\mathcal{D}_{\mathfrak{L}}$ of the following form:

$$
\mathcal{D}_{\mathfrak{L}}=\left.\mathrm{s}_{a}\right|_{b} ^{a} \mathrm{t}_{a}
$$

where the identity maps on $a$ and $b$ are implicit in this representation. Viewing $D_{b}^{\mathfrak{L}}$ and $G_{a}^{\mathfrak{L}}$ as topological spaces and $R_{a}^{\mathfrak{L}}$ as a continuous map (by "forgetting" their smooth structure), this data can be used to define a diagram in the category of topological spaces, i.e., a functor $\mathrm{X}^{\mathfrak{L}}: \mathcal{D}_{\mathfrak{L}} \rightarrow$ Top given by:

$$
\begin{gathered}
\mathbf{X}_{a}^{\mathfrak{L}}=G_{a}^{\mathfrak{L}} \\
\mathbf{X}^{\mathfrak{L}}\left(\mathcal{D}_{\mathfrak{L}}\right)=\quad \mathbf{X}_{\mathbf{s}_{a}}^{\mathfrak{L}}=\iota_{G_{a}}|| \mathbf{X}_{\mathfrak{t}_{a}}^{\mathfrak{L}}=R_{a}^{\mathfrak{L}} \\
\mathbf{X}_{b}^{\mathfrak{L}}=D_{b}^{\mathfrak{L}}
\end{gathered}
$$


This important observation is the motivation for the definition of a hybrid object over a category, e.g., the pair $\left(\mathcal{D}_{\mathfrak{L}}, \mathbf{X}^{\mathfrak{L}}\right)$ is a hybrid topological space. Before delving into these details, which will be introduced formally in the next section, we discuss some of the ramifications afforded by this alternative viewpoint.

Possibly the first question that arises naturally when studying hybrid topological spaces is:

\section{Can we understand hybrid topological spaces in terms of some "non-hybrid" counterpart?}

This question can and will be answered in the very general context of model categories, but first we discuss why problems arise if the question is addressed naively.

The most obvious way of associating a single topological space to a hybrid topological space is by the taking the colimit ${ }^{2}$

$$
\operatorname{colim}\left(\mathbf{X}^{\mathfrak{L}}\right)=\frac{D_{b}^{\mathfrak{L}}}{x \sim R_{a}^{\mathfrak{L}}(x), \quad x \in G_{a}^{\mathfrak{L}}} .
$$

In fact, this construction has been utilized in the context of hybrid systems [SJSL00] where it was referred to as the hybrifold. The problem with this construction is that it does not behave well "homotopically." Allen Hatcher describes this aptly in [Hat02]:

"It can easily happen that the [colimit] is rather useless because so much collapsing has occurred that little of the original diagram remains."

Homotopy theorists have long understood this problem (see [Vog73]). This motivated the introduction of the homotopy colimit as a method for obtaining a more "homotopy meaningful" topological space from a diagram of topological spaces. Homotopy colimits were first studied in the context of hybrid systems in [AS05], where it was shown that this space encodes useful information about the hybrid system, especially with respect to Zeno behavior, by studying the homology of the homotopy colimit of a hybrid space (for more on Zeno behavior see [Ame06a] and [ATS06]). The motivation for this work is to extend the use of homotopy colimits in the study of hybrid systems beyond the setting of topological spaces, i.e., to general categories that "admit a homotopy theory."

EXAMPLE 2.7. The hybrid topological space associated to the hybrid system modeling a bouncing ball, $\mathfrak{5}^{\text {ball }}$, is given by:

$$
\mathrm{X}^{\text {ball }}: \mathcal{D}_{\text {ball }} \rightarrow \text { Top, }
$$

where $\mathcal{D}_{\text {ball }}$ is the small category given in (2.3) and

$$
\begin{gathered}
\mathbf{X}_{a}^{\text {ball }}=G_{a}^{\text {ball }} \\
\mathbf{X}^{\text {ball }}\left(\mathcal{D}_{\text {ball }}\right)=\quad \mathbf{X}_{\mathrm{s}_{a}}^{\text {ball }}=\imath_{G_{a}^{\text {ball }}}||_{\nabla} \mathbf{X}_{\mathbf{t}_{a}}^{\text {ball }}=R_{a}^{\text {ball }} \\
\mathbf{X}_{b}^{\text {ball }}=D_{b}^{\text {ball }}
\end{gathered}
$$

A graphical representation of this hybrid topological space can be seen in Figure 3.

\footnotetext{
${ }^{2}$ This (slightly non-standard) notation means the following: " $x \sim R_{a}^{\mathfrak{L}}(x), \quad x \in G_{a}^{\mathfrak{L}}$ " is the least equivalence relation generated by the binary relation $\left(G_{a}^{\mathfrak{L}}, D_{b}^{\mathcal{L}}, \operatorname{Graph}\left(R_{a}^{\mathfrak{L}}\right)\right)$, with $\operatorname{Graph}\left(R_{a}^{\mathfrak{L}}\right) \subseteq G_{a}^{\mathfrak{L}} \times D_{b}^{\mathcal{L}}$ the graph of $R_{a}^{\mathfrak{L}}$, and $\frac{D_{b}^{\mathfrak{L}}}{x \sim R_{b}^{\mathfrak{L}}(x), x^{\prime} \in G_{a}^{\mathbb{L}}}$ is the corresponding quotient space.
} 


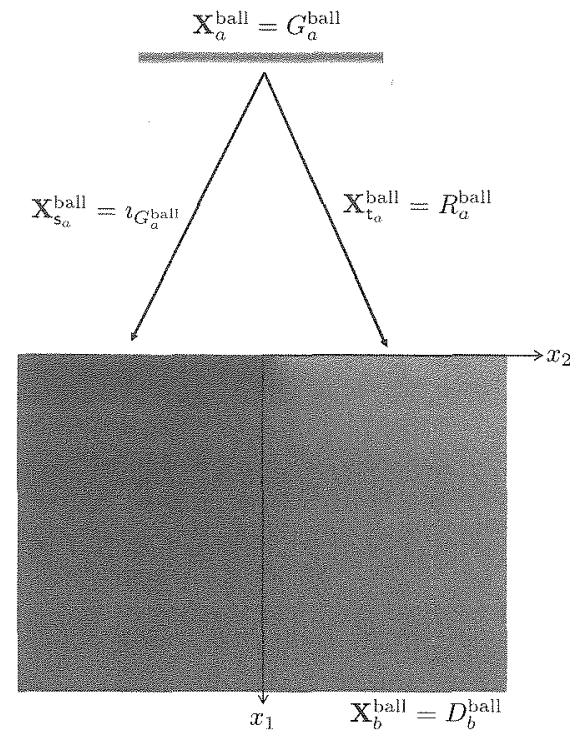

Figure 3. The hybrid topological space for the bouncing ball

\section{Hybrid Objects}

The starting point for introducing the notion of a hybrid object over a category is the observation that systems that display both contimuous and discrete behavior, i.e., hybrid systems, can be represented by a small category of a specific form, termed a $D$-category and denoted by $\mathcal{D}$, together with a functor:

$$
\mathrm{A}: \mathcal{D} \rightarrow \mathrm{C}
$$

where $C$ is the category of "non-hybrid" objects of interest. This alternative formulation of "hybrid objects" allows for the use of preexisting mathematical constructions in the study of hybrid systems, such as the one that defines a homotopy meaningful model structure on diagrams in a model category.

In this section we formally introduce D-categories and hybrid objects. Moreover, we demonstrate that the "standard" notion of a hybrid topological space corresponds to a hybrid object over the category of topological spaces. A more detailed discussion of hybrid systems, hybrid objects, and their relationship can be found in [Ame06a]. We refer the reader to [Lan98] for additional background information on category theory.

3.1. D-categories. Let $\mathcal{D}$ be a small category. We use $\operatorname{Mor}(\mathcal{D})$ to denote the morphisms of $\mathcal{D}$, i.e.,

$$
\operatorname{Mor}(\mathcal{D})=\bigcup_{(a, b) \in \operatorname{Ob}(\mathcal{D}) \times \operatorname{Ob}(\mathcal{D})} \operatorname{Hom}_{\mathcal{D}}(a, b)
$$

and $\operatorname{Mor}_{i}(\mathcal{D})$ to denote the set of non-identity morphisms of $\mathcal{D}$, i.e.,

$$
\operatorname{Mor}_{\text {id }}(\mathcal{D})=\{\alpha \in \operatorname{Mor}(\mathcal{D}): \alpha \neq \mathrm{id}\} .
$$

For a morphism $\alpha: a \rightarrow b$ in $\mathcal{D}$, its domain is denoted by $\operatorname{dom}(\alpha)=a$ and its codomain is denoted by $\operatorname{cod}(\alpha)=b$. 
Definition 3.1. A $D$-category is a small category $\mathcal{D}$ such that:

- There exist two subsets of $\mathrm{Ob}(\mathcal{D}), \mathrm{E}(\mathcal{D})$ and $\mathrm{V}(\mathcal{D})$, termed the edge set and the vertex set, satisfying:

$$
\begin{aligned}
& \mathrm{E}(\mathcal{D}) \cap \mathrm{V}(\mathcal{D})=\emptyset, \\
& \mathrm{E}(\mathcal{D}) \cup \mathrm{V}(\mathcal{D})=\mathrm{Ob}(\mathcal{D}),
\end{aligned}
$$

- There exists a pair of functions:

$$
\mathrm{E}(\mathcal{D}) \stackrel{\mathrm{s}}{\mathrm{t}} \operatorname{Mor}_{\text {id }}(\mathcal{D}),
$$

such that:

$$
\begin{aligned}
& \mathrm{s}(\mathrm{E}(\mathcal{D})) \cap \mathrm{t}(\mathrm{E}(\mathcal{D}))=\emptyset \\
& \mathrm{s}(\mathrm{E}(\mathcal{D})) \cup \mathrm{t}(\mathrm{E}(\mathcal{D}))=\operatorname{Mor}_{\text {id }}(\mathcal{D}) .
\end{aligned}
$$

The pair $(\mathrm{s}, \mathrm{t})$ is termed an orientation of $\mathcal{D}$.

- The following diagram:

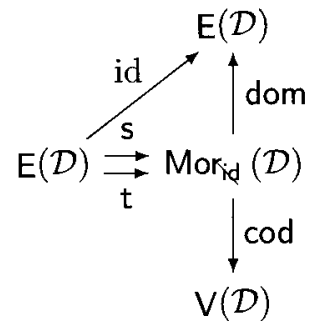

commutes.

The definition of a D-category $\mathcal{D}$ implies that for every $a \in \mathrm{E}(\mathcal{D})$, there is a diagram of the form

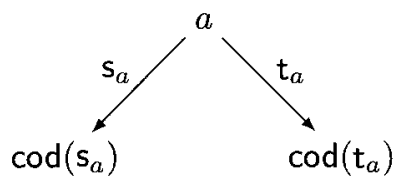

where $\operatorname{cod}\left(\mathbf{s}_{a}\right), \operatorname{cod}\left(\mathbf{t}_{a}\right) \in \mathrm{V}(\mathcal{D})$. Diagrams of this form can be thought of as the "canonical" D-categories-note the similarity between this diagram and the one given in (2.3).

3.2. D-categories and graphs. D-categories can be essentially thought of as graphs (although, in the context of hybrid systems, it is not sufficient to work with graphs). That is, to every D-category there is an associated graph and, conversely, to every graph there is an associated D-category.

More specifically, given a graph $\Gamma=(Q, E)$, one associates to this graph a D-category $\mathcal{D}_{\Gamma}$ by defining the edge and vertex sets, and hence the objects, to be:

$$
\mathrm{E}\left(\mathcal{D}_{\Gamma}\right):=E, \quad \mathrm{~V}\left(\mathcal{D}_{\Gamma}\right):=Q, \quad \mathrm{Ob}\left(\mathcal{D}_{\Gamma}\right)=\mathrm{E}\left(\mathcal{D}_{\Gamma}\right) \cup \mathrm{V}\left(\mathcal{D}_{\Gamma}\right)
$$

To define the orientation $(\mathrm{s}, \mathrm{t})$ of $\mathcal{D}_{\Gamma}$ we define, for every $e \in E$, morphisms:

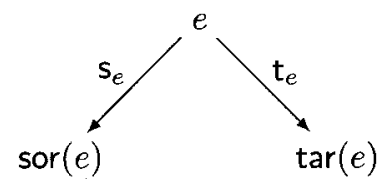


We complete the description of $\mathcal{D}_{\Gamma}$ by defining an identity morphism on each object of $\mathcal{D}_{\Gamma}$.

Conversely, one can associate to a D-category $\mathcal{D}$ a graph

$$
\Gamma_{\mathcal{D}}:=(\mathrm{V}(\mathcal{D}), \mathrm{E}(\mathcal{D}))
$$

with source and target maps given by:

$$
\mathrm{E}(\mathcal{D}) \frac{\text { sor }=\operatorname{cod}\left(\mathrm{s}_{(-)}\right)}{\underset{\operatorname{tar}=\operatorname{cod}\left(\mathrm{t}_{(-)}\right)}{\longrightarrow}} \mathrm{V}(\mathcal{D})
$$

More generally, it was shown in [Ame06a] that Dcat $\cong$ Grph, where Grph is the category of graphs (as defined in [Lan98]) and Dcat is the category of Dcategories which has as objects D-categories and morphisms functors that "preserve orientations."

EXAMPLE 3.2. The D-category obtained from the graph $\Gamma^{\mathfrak{L}}$ given in $(2.1)$ is the D-category $\mathcal{D}_{\mathfrak{L}}$ given in (2.3) and, conversely, the graph obtained from the D-category $\mathcal{D}_{\mathfrak{L}}$ is the graph $\Gamma^{\mathfrak{L}}$.

3.3. Hybrid Objects. With the notion of a D-category in hand, we define hybrid objects over a category $C$.

DEFINITION 3.3. Let $C$ be a category. A hybrid object over $C$ is a D-category $\mathcal{D}$ together with a functor

$$
\mathbf{A}: \mathcal{D} \rightarrow \mathrm{C}
$$

The functor $\mathbf{A}$ can be thought of as the continuous component of the hybrid object, and the category $\mathcal{D}$ as its discrete component. The category $\mathrm{C}^{\mathcal{D}}$ is thus the category of hybrid objects over $C$ with the same "discrete structure." That is, the objects of this category are pairs $(\mathcal{D}, \mathbf{A}),(\mathcal{D}, \mathbf{B}), \ldots$, and the morphisms between two objects of $\mathrm{C}^{\mathcal{D}},(\mathcal{D}, \mathbf{A})$ and $(\mathcal{D}, \mathbf{B})$, are natural transformations $\vec{f}: \mathbf{A} \stackrel{\circ}{\rightarrow}$.

EXAMPLE 3.4. Some specific examples of hybrid objects are given by:
Hybrid simplicial set
$\mathbf{K}: \mathcal{D} \rightarrow$ SSet
Hybrid chain complex
$\mathrm{C}: \mathcal{D} \rightarrow \mathrm{Ch}(\mathrm{A})$
Hybrid manifold
$\mathbf{M}: \mathcal{D} \rightarrow$ Man

where SSet is the category of simplicial sets, $\mathrm{Ch}(\mathrm{A})$ is the category of chain complexes over an abelian category $A$ and Man is the category of (smooth) manifolds.

More generally, we will be interested in studying hybrid objects over a model category $\mathrm{M}$, i.e., functors $\mathbf{A}: \mathcal{D} \rightarrow M$.

REMARK 3.5. Utilizing the category of D-categories, Dcat, one can define the category of hybrid objects over a category $C$, denoted by $\mathrm{Hy}(\mathrm{C})$. The objects of this category are hybrid objects over $C$, i.e., pairs $(\mathcal{A}, \mathbf{A}),(\mathcal{B}, \mathbf{B}), \ldots$, with $\mathcal{A}$ and $\mathcal{B}$ D-categories and $\mathbf{A}: \mathcal{A} \rightarrow \mathbf{C}$ and $\mathbf{B}: \mathcal{B} \rightarrow C$ functors. The morphisms are pairs $(\vec{F}, \vec{f}):(\mathcal{A}, \mathbf{A}) \rightarrow(\mathcal{B}, \mathbf{B})$, where $\vec{F}: \mathcal{A} \rightarrow \mathcal{B}$ is a morphism in Dcat and $\vec{f}: \mathbf{A} \dot{\rightarrow} \mathbf{B} \circ \vec{F}$ is a morphism in $\mathbf{C}^{\mathcal{A}}$.

In this paper, we will not consider explicitly the category $\mathrm{Hy}(\mathrm{C})$, but properties of this category have been studied in both [Ame06a] and [Ame06b]. 
3.4. Hybrid topological spaces. We justify the notion of a hybrid object by relating hybrid topological spaces to hybrid spaces (see Definition 2.6).

A hybrid topological space is given by a pair $(\mathcal{D}, \mathbf{X})$, where $\mathbf{X}: \mathcal{D} \rightarrow$ Top. In physical systems such as robotic systems, it often is the case that for every $a \in E(\mathcal{D})$, and hence every diagram in $\mathcal{D}$ of the form given in (3.1), the corresponding diagram in Top is given by:

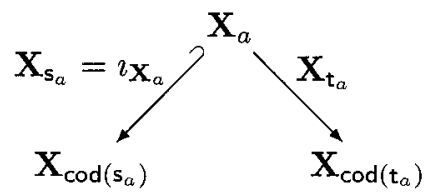

where $\mathbf{X}_{a} \subseteq \mathbf{X}_{\operatorname{cod}\left(\mathbf{s}_{a}\right)}$ is a subspace of $\mathbf{X}_{\operatorname{cod}\left(\mathbf{s}_{a}\right)}$ and $\mathbf{X}_{\mathbf{s}_{a}}=\imath \mathbf{x}_{a}$ is the natural inclusion. We denote hybrid topological spaces of this form by $\mathbf{X}^{2}$.

Although we do not assume explicitly that $\mathbf{X}_{\mathbf{s}_{a}}$ is an inclusion, this often is the case, as the following proposition indicates.

Proposition 3.6. There is a bijective correspondence:

$$
\begin{gathered}
\text { \{Hybrid Spaces, } \mathbb{H}=(\Gamma, D, G, R)\} \\
\left\{\text { Hybrid Topological Spaces, } \mathbf{X}^{\imath}: \mathcal{D} \rightarrow \text { Top }\right\} .
\end{gathered}
$$

Proof. Given a hybrid space $\mathbb{H}=(\Gamma, D, G, R)$, we define the corresponding hybrid topological space to be $\mathbf{X}^{(D, G, R)}: \mathcal{D}_{\Gamma} \rightarrow$ Top, where $\mathcal{D}_{\Gamma}$ is the D-category obtained from the graph $\Gamma$ as described in Paragraph 3.2 and $\mathbf{X}^{(D, G, R)}$ is defined for every $e \in \mathrm{E}\left(\mathcal{D}_{\Gamma}\right)=E$ by

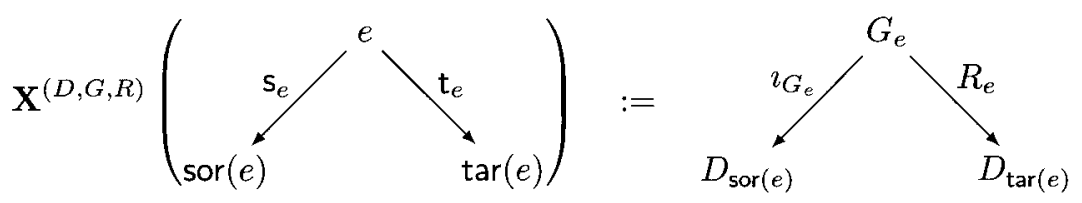

It is clear that $\mathbf{X}^{(D, G, R)}: \mathcal{D}_{\Gamma} \rightarrow$ Top is a hybrid topological space.

Conversely, consider a hybrid topological space $\mathbf{X}^{\imath}: \mathcal{D} \rightarrow$ Top. Let $\Gamma_{\mathcal{D}}=$ $(\mathrm{V}(\mathcal{D}), \mathrm{E}(\mathcal{D}))$ be the graph obtained from the D-category $\mathcal{D}$ as defined in Paragraph 3.2. We define $\mathbb{H}_{\left(\mathcal{D}, \mathbf{X}^{2}\right)}=\left(\Gamma_{\mathcal{D}}, D_{\mathbf{X}^{2}}, G_{\mathbf{X}^{2}}, R_{\mathbf{X}^{2}}\right)$, where $D_{\mathbf{X}^{2}}:=\left\{\mathbf{X}_{b}^{\imath}\right\}_{b \in \mathrm{V}(\mathcal{D})}, G_{\mathbf{X}^{2}}:=$ $\left\{\mathbf{X}_{a}^{\imath}\right\}_{a \in \mathrm{E}(\mathcal{D})}$ and $R_{\mathbf{X}^{\imath}}:=\left\{\mathbf{X}_{\mathbf{t}_{a}}^{\imath}\right\}_{a \in \mathrm{E}(\mathcal{D})}$.

\section{Model Categories}

This section introduces the basics of model categories. While these concepts can be found in many references ([Qui67], [CS02], [DHKS04], [Hov99] and [DS95], to name a few), we briefly revisit them here in order to justify subsequent constructions. We begin by recalling the definition of a model category. Note that this definition of a model category essentially corresponds to the notion of a closed model category as introduced by Quillen [Qui67].

4.1. Model categories. A model category $M$ is a category with three special classes of morphisms:

- weak equivalences (denoted by $\stackrel{\sim}{\longrightarrow}$ ),

- fibrations (denoted by $\longrightarrow$ ),

- cofibrations (denoted by $\longrightarrow$ ), 
which are closed under composition and contain all identity morphisms. In addition, they must satisfy axioms MC1, MC2, MC3, MC4 and MC5 as given in [DS95] (Definition 3.3), except that we strengthen ${ }^{3} \mathbf{M C 1}$ by assuming that $\mathrm{M}$ is complete and cocomplete, i.e., small limits and colimits exist in $M$. These axioms simply give natural conditions on the relationships between weak equivalences, fibrations and cofibrations. For example, MC5 states that any morphism $f: A \rightarrow B$ can be factored in the following two ways: (1) $f=p \circ i$ where $i$ is a cofibration and $p$ is an acyclic fibration, (2) $f=p \circ i$ where $i$ is an acyclic cofibration and $p$ is a fibration.

4.2. A model structure on Top. Since we are interested in studying the topological properties of hybrid systems, we will consider the category of topological spaces, Top. This category provides an example of a model category (as one would expect). What is interesting is that there is not a unique model structure on Top. This is common when dealing with categories that admit model structures - these structures often are not unique, so the specific model structure chosen depends on the application. In our case, we will consider the model structure in which the weak equivalences are homotopy equivalences following the excellent paper by Strøm [Str72].

A morphism $p: X \rightarrow Y$ in Top is a Hurewicz fibration if it has the homotopy lifting property with respect to all topological spaces, i.e., for every topological space $A$ such that the solid arrows in the following diagram commute

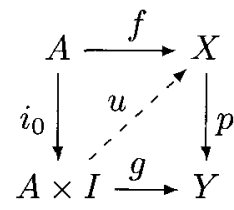

where $I$ is the unit interval and $i_{0}(a)=(a, 0)$, there exists a $u: A \times I \rightarrow X$ making the entire diagram commute.

Let $X$ be a closed subspace of $Y$. The inclusion $i: X \rightarrow Y$ is a (closed) Hurewicz cofibration if it has the homotopy extension property with respect to all topological spaces, i.e., for every topological space $Z$ such that the solid arrows in the following diagram commute

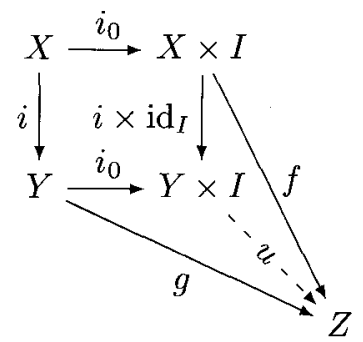

there exists a $u: Y \times I \rightarrow Z$ making the entire diagram commute.

With these formulations, the category of topological spaces has the following model structure:

\footnotetext{
${ }^{3}$ The motivation for strengthening MC1 is that we want to allow for the possibility of Dcategories with an infinite number of objects.
} 
THEOREM 4.1 ([Str72]). The category of topological spaces, Top, is a model category with the following choices of weak equivalences, fibrations and cofibrations: a morphism $f: X \rightarrow Y$ is

We: $A$ weak equivalence if it is a homotopy equivalence,

Cof: A cofibration if it is a (closed) Hurewicz cofibration,

Fib: A fibration if it is a Hurewicz fibration.

4.3. Cofibrant and fibrant objects. An object $A$ of $\mathrm{M}$ is said to be cofibrant if the morphism from the initial object of $M$ to $A$ is a cofibration: $\emptyset \longrightarrow A$. An object $B$ of $\mathrm{M}$ is fibrant if the morphism from $B$ to the terminal object is a fibration: $B \longrightarrow *$.

Given an object $A$ of $\mathrm{M}$, we can use MC5(1) to define its cofibrant replacement. That is, for the morphism $\emptyset \rightarrow A$, there is a factorization:

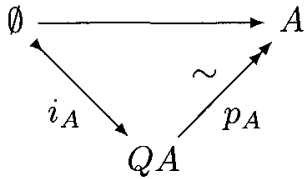

Therefore, $Q A$ is a cofibrant object weakly equivalent to $A$, termed the cofibrant replacement of $A$.

Similarly, for an object $B$ of $\mathrm{M}$ and the morphism $B \rightarrow *$, there is a factorization:

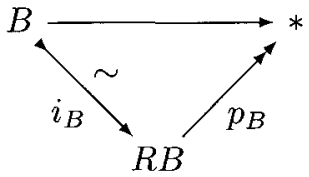

Therefore, $R B$ is a fibrant object weakly equivalent to $B$, termed the fibrant replacement of $B$.

Fibrant and cofibrant replacements are functorial in the following sense: for every morphism $f: A \rightarrow B$ there exist morphisms $Q f: Q A \rightarrow Q B$ and $R f: R A \rightarrow$ $R B$ such that there is a commuting diagram:

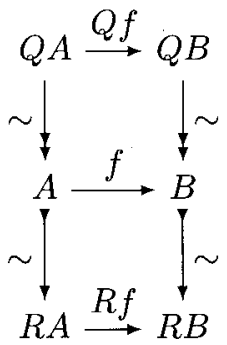

Moreover, $f$ is a weak equivalence iff $Q f$ is a weak equivalence iff $R f$ is a weak equivalence.

EXAMPLE 4.2. For the category of topological spaces, every object is both cofibrant and fibrant. This implies that the cofibrant and fibrant replacement of a topological space can be taken to be the original topological space. 
4.4. Left homotopies. Consider the morphism 〈id, id〉 : $A \amalg A \rightarrow A$. A cylinder object, denoted by $\operatorname{Cyl}(A)$, is any object such that there is a factorization:

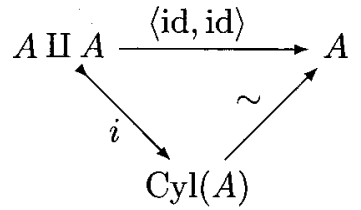

Cylinder objects exist by MC5(1). A left homotopy from $f: A \rightarrow B$ to $g: A \rightarrow B$ is a morphism $H: \operatorname{Cyl}(A) \rightarrow B$ making the following diagram

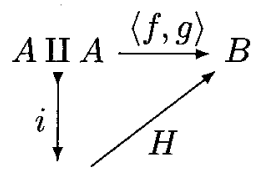

$$
\operatorname{Cyl}(A)
$$

commute. In this case, we write $f \stackrel{l}{\simeq} g$.

There is also the notion of a right homotopy from $f: A \rightarrow B$ to $g: A \rightarrow$ $B$, denoted by $f \stackrel{r}{\simeq} g$, but since $f \stackrel{l}{\simeq} g$ iff $f \stackrel{r}{\simeq} g$ when $A$ is cofibrant and $B$ is fibrant (Lemma 4.21, [DS95]), we forgo introducing right homotopies for the sake of brevity. In addition, as a result of this observation, when $A$ and $B$ are both fibrant and cofibrant, if $f \stackrel{l}{\simeq} g$ we say that $f$ and $g$ are homotopic and write $f \simeq g$.

EXAMPLE 4.3. In the category of topological spaces, Top, $X \times I$ is a cylinder object. The map $i: X \amalg X \rightarrow X \times I$ is given by $i=\left\langle i_{0}, i_{1}\right\rangle$, where $i_{0}(x)=(x, 0)$ and $i_{1}(x)=(x, 1)$. Two maps $f, g: X \rightarrow Y$ between topological spaces are left homotopic iff they are homotopic in the traditional sense.

4.5. The homotopy category. Let $A$ and $B$ be objects of $\mathrm{M}$ that are both fibrant and cofibrant. Then the binary relation on $\operatorname{Hom}_{\mathrm{M}}(A, B)$ given by associating homotopic morphisms forms an equivalence relation: $\sim$. Therefore, define

$$
\pi(A, B)=\operatorname{Hom}_{\mathrm{M}}(A, B) / \sim
$$

where $[f] \in \pi(A, B)$ is given by $[f]=\left\{g \in \operatorname{Hom}_{\mathrm{M}}(A, B): f \simeq g\right\}$.

Since the fibrant-cofibrant replacement $R Q A$ of an object of $A$ is simultaneously fibrant and cofibrant, consider the following:

Definition 4.4. The homotopy category $\mathrm{Ho}(\mathrm{M})$ of a model category $\mathrm{M}$ is a category with the same objects as $\mathrm{M}$ and with

$$
\mathrm{Hom}_{\mathrm{Ho}(\mathrm{M})}(A, B)=\pi(R Q A, R Q B) .
$$

Recall that for all morphisms $f: A \rightarrow B$ ( $A$ and $B$ arbitrary objects of $\mathrm{M}$ ), we have a commuting diagram:

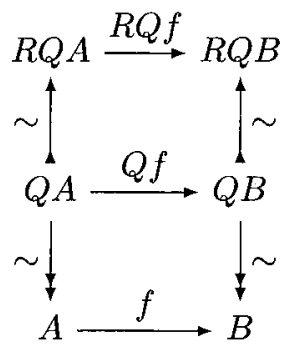


Therefore, we can define a functor $\gamma: \mathrm{M} \rightarrow \mathrm{Ho}(\mathrm{M})$ with $\gamma(A)=A$ for all objects of $A$ of $\mathrm{M}$ and $\gamma(f)=[R Q f]$. (See [Qui67] for more on the structure of homotopy categories.)

The following lemma (see [Qui67]) relates homotopies with weak equivalences:

LEMMA 4.5. If $f: A \rightarrow B$ with $A$ and $B$ both fibrant and cofibrant, then $f$ is a weak equivalence iff $f$ is homotopic to the identity, i.e., there exists a morphism $g: B \rightarrow A$ such that $f \circ g \simeq \mathrm{id}_{B}$ and $g \circ f \simeq \mathrm{id}_{A}$. Therefore, $f$ is a weak equivalence in $\mathrm{M}$ iff $\gamma(f)$ is an isomorphism in $\mathrm{Ho}(\mathrm{M})$.

EXAMPLE 4.6. Since every topological space is both fibrant and cofibrant, the homotopy category of Top, Ho(Top), is the traditional homotopy category obtained by formally inverting homotopy equivalences.

\section{Homotopy Meaningful Model Structures}

We now introduce the notion of a Quillen adjunction, which is fundamental in understanding the interplay among different model categories. This follows from the fact that adjunctions of this form imply the existence of total (left and right) derived functors and thus induce an adjunction between homotopy categories. The discussion of homotopy colimits utilizes this observation in a fundamental fashion. Again, the contents of this section can be found in many references - most notably [DS95], [DHKS04] and [Qui67]. They are reintroduced here so as to justify the notion of a homotopy meaningful model category structure and the specific model structure that is chosen for $\mathrm{M}^{\mathcal{D}}$.

To motivate the introduction of derived functors, and hence Quillen adjunctions, consider a functor $F: M \rightarrow D$ with $\mathrm{M}$ a model category. In general, this functor does not factor through the homotopy category of $M$, i.e., there does not exist a factorization:

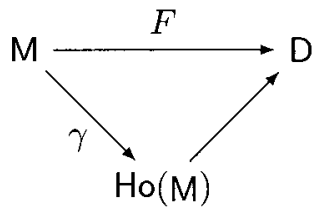

Left and right derived functors are introduced in order to find the "closest approximation" to such a factorization "from the left" or "from the right."

5.1. Left derived functors. Let $F: \mathrm{M} \rightarrow \mathrm{D}$ be a functor, with $\mathrm{M}$ a model category. The left derived functor of $F$ is a functor $L F: \mathrm{Ho}(\mathrm{M}) \rightarrow \mathrm{D}$ together with a natural transformation $t: L F \circ \gamma \dot{\rightarrow} F$ where, again, $\gamma: \mathrm{M} \rightarrow \mathrm{Ho}(\mathrm{M})$. In addition, it must satisfy the universal property that for any $G: \mathrm{Ho}(\mathrm{M}) \rightarrow \mathrm{D}$ and any $s: G$ o $\gamma \dot{\rightarrow} F$ there exists a unique $s^{\prime}: G \dot{\rightarrow} L F$ such that the following diagram

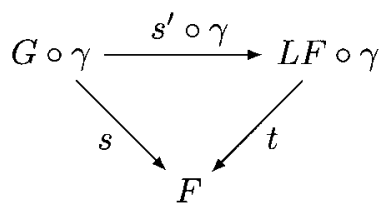

commutes. While the left derived functor of a functor is not unique, it is unique up to isomorphism. 
The total left derived functor of a functor $F: M \rightarrow N$ between model categories is a functor

$$
\mathbb{L} F: \mathrm{Ho}(\mathrm{M}) \rightarrow \mathrm{Ho}(\mathrm{N})
$$

such that $\mathbb{L} F$ is the left derived functor of the composite $\gamma_{N} \circ F: M \rightarrow H o(N)$, where $\gamma_{\mathrm{N}}: \mathrm{N} \rightarrow \mathrm{Ho}(\mathrm{N})$.

Right derived functors and total right derived functors are defined dually. In particular, the total right derived functor of a functor $F: \mathrm{M} \rightarrow \mathrm{N}$ between model categories is a functor $\mathbb{R} F: \mathrm{Ho}(M) \rightarrow \mathrm{Ho}(\mathrm{N})$.

The following result is very useful:

Proposition 5.1 ([Qui67]). Let $F: \mathrm{M} \rightarrow \mathrm{D}$ with $\mathrm{M}$ a model category. If $F(f)$ is an isomorphism whenever $f$ is a weak equivalence between cofibrant objects, then the left derived functor $L F$ of $F$ exists and

$$
L F(A) \cong F(A)
$$

for every cofibrant object $A$ of $\mathrm{M}$.

This motivates the following (where the terminology is chosen based upon Definition 3.3 of $[\mathbf{C S 0 2}]$ ):

Definition 5.2. A functor $F: \mathrm{M} \rightarrow \mathrm{N}$ between model categories is said to be cofibrantly homotopy meaningful if it preserves weak equivalences between cofibrant objects, i.e., if $F(f)$ is a weak equivalence whenever $f$ is a weak equivalence between cofibrant objects.

The importance of homotopy meaningful functors is outlined in the following straightforward corollary of Proposition 5.1, which is essential when considering homotopy colimits.

Corollary 5.3. If $F: \mathrm{M} \rightarrow \mathrm{N}$ is cofibrantly homotopy meaningful, then the total left derived functor $\mathbb{L} F: \mathrm{Ho}(\mathrm{M}) \rightarrow \mathrm{Ho}(\mathrm{N})$ exists and can be computed by:

$$
\mathbb{L} F(A) \cong F\left(A^{\prime}\right)
$$

for any cofibrant object $A^{\prime}$ weakly equivalent to $A$.

In particular, this corollary implies that if $Q A$ is a cofibrant replacement of $A$, then $\mathbb{L} F(A) \cong F(Q A)$.

5.2. Quillen Adjunctions. Let $M$ and $N$ be model categories and let

$$
F: \mathrm{M} \rightleftarrows \mathrm{N}: G
$$

be an adjunction. This adjunction is a Quillen adjunction if

(i) $F$ preserves cofibrations and acyclic cofibrations,

(ii) $G$ preserves fibrations and acyclic fibrations.

It is easy to verify that (i) and (ii) are equivalent. Therefore, Quillen adjunctions are intrinsically related to the existance of left and right derived functors as the following proposition indicates.

Proposition 5.4 ([Qui67]). Let $\mathrm{M}$ and $\mathrm{N}$ be model categories, and

$$
F: \mathrm{M} \rightleftarrows \mathrm{N}: G
$$

be a Quillen adjunction. Then the total derived functors:

$$
\mathbb{L} F: \mathrm{Ho}(\mathrm{M}) \rightleftarrows \mathrm{Ho}(\mathrm{N}): \mathbb{R} G
$$


exist and form an adjunction.

Related to this proposition (and the proof thereof, see [DS95]) is the following lemma due to $\mathrm{K}$. Brown, which is useful in its own right.

LEMMA 5.5 (Brown's Lemma). Let $F: \mathrm{M} \rightarrow \mathrm{N}$ be a functor between model categories. If $F$ carries acyclic cofibrations to weak equivalences, then $F$ is cofibrantly homotopy meaningful.

5.3. Homotopy Meaningful Model Category Structures. Let M denote a model category and $J$ a small category. The goal is to give conditions on the model structure of $\mathrm{M}^{\mathrm{J}}$, if such a structure exists, so that it is cofibrantly homotopy meaningful, i.e., a model structure such that the total left derived functor of colim exists. More formally, consider the following:

DEFINITION 5.6. A model category structure on $\mathrm{M}^{\rfloor}$is said to be cofibrantly homotopy meaningful if colim is cofibrantly homotopy meaningful, i.e., if colim preserves weak equivalences between cofibrant objects.

Since there is an adjunction:

$$
\text { colim : } \mathrm{M}^{\mathrm{J}} \rightleftarrows \mathrm{M}: \Delta,
$$

with $\Delta$ the constant functor, for a model category structure on $\mathrm{M}^{\lrcorner}$to be cofibrantly homotopy meaningful, we need this adjunction to be a Quillen adjunction, i.e., we need $\Delta$ to preserve fibrations and acyclic fibrations. This helps to characterize cofibrantly homotopy meaningful model structures on $\mathrm{M}^{J}$. That is:

PROPOSITION 5.7. If there exists a model structure on $\mathrm{M}^{\mathrm{J}}$, then it is cofibrantly homotopy meaningful if:

(i) The weak equivalences are objectwise weak equivalences in $\mathrm{M}$,

(ii) The fibrations are objectwise fibrations in $\mathrm{M}$.

In this case, the total left derived functor of colim exists and is termed the homotopy colimit:

Moreover,

$$
\text { hocolim := Lcolim }: \mathrm{Ho}\left(\mathrm{M}^{\lrcorner}\right) \rightarrow \mathrm{Ho}(\mathrm{M})
$$

$$
\operatorname{hocolim}(A) \cong \operatorname{colim}\left(A^{\prime}\right)
$$

for any cofibrant object $A^{\prime}$ weakly equivalent to $A$.

PROOF. This result follows in a straightforward manner from the other results mentioned in this section. By (i) and (ii) we know that $\Delta$ preserves fibrations and acyclic fibrations, and so the adjunction (5.1) is a Quillen adjunction. Therefore, hocolim exists by Proposition 5.4. Now, by Brown's Lemma, colim is cofibrantly homotopy meaningful (again, since (5.1) is a Quillen adjunction). Finally, by Corollary 5.3, it follows that hocolim $(A) \cong \operatorname{colim}\left(A^{\prime}\right)$ for any cofibrant object $A^{\prime}$ weakly equivalent to $A$.

\section{Hybrid Model Structures}

Given a model category $M$ and a $D$-category $\mathcal{D}$, in this section we determine a cofibrantly homotopy meaningful model structure on $M^{\mathcal{D}}$. To illustrate the concepts introduced, we construct homotopy pushouts in general model categories which, to provide a concrete example, will be specialized to the category of topological spaces. 
6.1. Setup. We begin by introducing some constructions necessary for the main result of this paper.

For $b \in \mathrm{V}(\mathcal{D})$, define

$$
\begin{aligned}
\mathrm{E}(\mathcal{D})_{b} & =\{a \in \mathrm{E}(\mathcal{D}): \exists \alpha: a \rightarrow b \text { in } \mathcal{D}\} \\
& =\left\{a \in \mathrm{E}(\mathcal{D}): b=\operatorname{cod}\left(\mathbf{s}_{a}\right) \text { or } b=\operatorname{cod}\left(\mathbf{t}_{a}\right)\right\} .
\end{aligned}
$$

For example, if the subcategory of $\mathcal{D}$ of all morphisms with codomain $b$ has the general form:

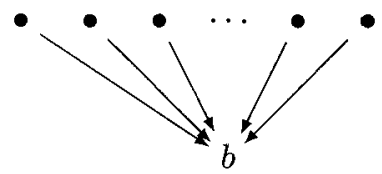

the set $\mathrm{E}(\mathcal{D})_{b}$ consists of all of the objects on the top of this diagram, which is necessarily a subset of $E(\mathcal{D})$.

Let $\vec{f}: \mathbf{A} \dot{\rightarrow} \mathbf{B}$ in $\mathrm{M}^{\mathcal{D}}$. Consider the following morphisms:

- For every $a \in \mathrm{E}(\mathcal{D})$, define $i_{a}(\vec{f})=\vec{f}_{a}$.

- For every $b \in \mathrm{V}(\mathcal{D})$, define $i_{b}(\vec{f})$ to be the unique morphism induced by the following pushout diagram:

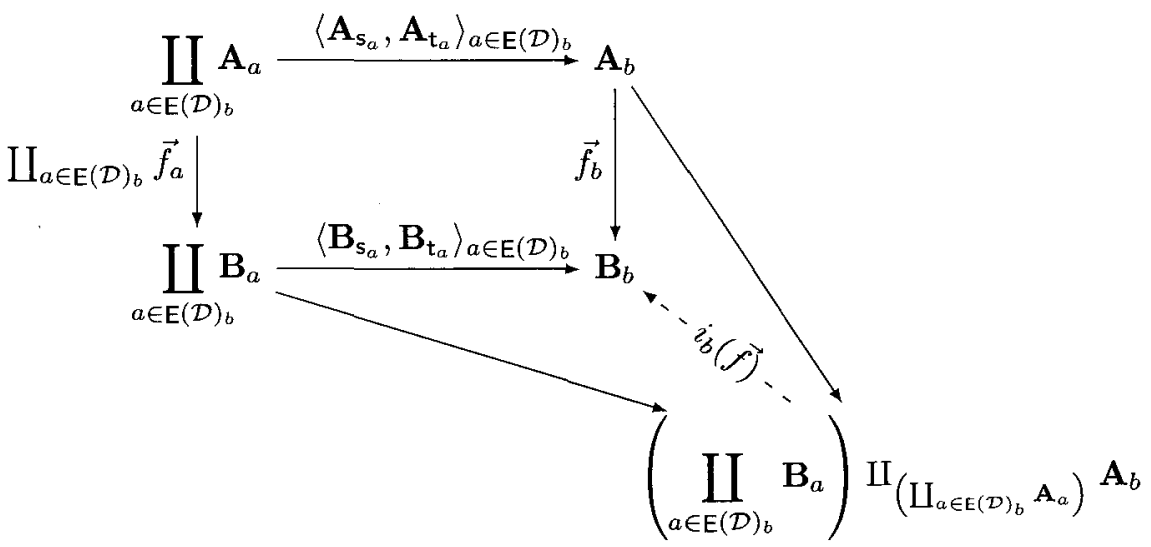

where $\left\langle\mathbf{A}_{\mathbf{s}_{a}}, \mathbf{A}_{\mathbf{t}_{a}}\right\rangle_{a \in \mathrm{E}(\mathcal{D})_{b}}$ and $\left\langle\mathbf{B}_{\mathbf{s}_{a}}, \mathbf{B}_{\mathbf{t}_{a}}\right\rangle_{a \in \mathrm{E}(\mathcal{D})_{b}}$ are the unique morphisms induced by the coproduct; for example, $\left\langle\mathbf{A}_{\mathbf{s}_{a}}, \mathbf{A}_{\mathbf{t}_{a}}\right\rangle_{a \in \mathrm{E}(\mathcal{D})_{b}}$ is the unique morphism making the following diagram commute:

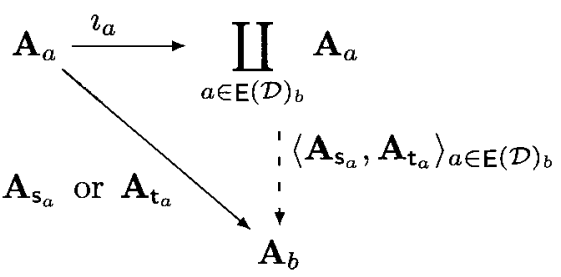

Using these definitions, we present the main result of this paper.

THEOREM 6.1. For any $D$-category $\mathcal{D}$ and model category $\mathrm{M}$, the category $\mathrm{M}^{\mathcal{D}}$ is a cofibrantly homotopy meaningful model category for the following choices of weak equivalences, fibrations and cofibrations: a morphism $\vec{f}: \mathbf{A} \dot{\rightarrow} \mathbf{B}$ is

We: $A$ weak equivalence if $\vec{f}$ is objectwise a weak equivalence in $\mathrm{M}$, i.e., $\vec{f}_{a}$ is a weak equivalence for all $a \in \mathrm{Ob}(\mathcal{D})$, 
Fib: $A$ fibration if $\vec{f}$ is objectwise a fibration in $\mathrm{M}$,

Cof: $A$ cofibration if $i_{a}(\vec{f})$ and $i_{b}(\vec{f})$ are cofibrations in $\mathrm{M}$ for all $a \in \mathrm{E}(\mathcal{D})$ and $b \in \mathrm{V}(\mathcal{D})$

This theorem is simply a corollary of a more general theorem relating to the model structure of diagrams over direct categories. Therefore, proving the theorem amounts to discussing how it fits within this more general framework - that is, the main result of this paper is not the theorem per se, but rather the observations that hybrid objects can be represented categorically and that this representation is such that preexisting results can be utilized.

ProOF. Recall that the category 2 consists of two objects and a single (nonidentity) morphism: $0 \rightarrow 1$. Define the degree functor deg : $\mathcal{D} \rightarrow \mathbf{2}$ on objects $a \in \mathcal{D}$ by

$$
\operatorname{deg}(a)=\left\{\begin{array}{lll}
0 & \text { if } & a \in \mathrm{E}(\mathcal{D}) \\
1 & \text { if } & a \in \mathrm{V}(\mathcal{D})
\end{array}\right.
$$

This functor sends every (non-identity) morphism in $\mathcal{D}$ to the single (non-identity) morphism in $\mathbf{2}$ by the definition of a D-category. Since deg is thus a linear extension, every $\mathrm{D}$-category $\mathcal{D}$ is a direct category. The theorem now follows from Theorem 5.1.3 in [Hov99]; for a more thorough explanation, see [Ame06b].

The importance of Theorem 6.1 is that the model structure on $M^{\mathcal{D}}$ was defined in such a way that homotopy colimits exist, thus relating the model structure on $\mathrm{M}^{\mathcal{D}}$ to the model structure on $\mathrm{M}$. That is, we have the following:

Corollary 6.2. For every model category $\mathrm{M}$ and $D$-category $\mathcal{D}$,

$$
\text { hocolim }: \mathrm{Ho}\left(\mathrm{M}^{\mathcal{D}}\right) \rightarrow \mathrm{Ho}(\mathrm{M})
$$

exists and

- hocolim $(\mathbf{A}) \cong \operatorname{hocolim}(\mathbf{B})$ if $\mathbf{A}$ and $\mathbf{B}$ are weakly equivalent.

- $\operatorname{hocolim}(\mathbf{A}) \cong \operatorname{colim}\left(\mathbf{A}^{\prime}\right)$ for every cofibrant hybrid object $\mathbf{A}^{\prime}$ weakly equivalent to $\mathbf{A}$.

Proof. Follows from Theorem 6.1 and Proposition 5.7.

This corollary indicates that an important aspect of computing homotopy colimits is understanding what the cofibrant objects are in $M^{\mathcal{D}}$. This motivates the final result of this paper.

Proposition 6.3. For every D-category $\mathcal{D}$, an object $\mathbf{A}: \mathcal{D} \rightarrow \mathbf{M}$ of $\mathrm{M}^{\mathcal{D}}$ is cofibrant if for every $a \in \mathrm{E}(\mathcal{D})$ :

- $\mathbf{A}_{a}$ is a cofibrant object of $\mathrm{M}$,

- $\mathbf{A}_{\mathbf{s}_{a}}$ and $\mathbf{A}_{\mathbf{t}_{a}}$ are cofibrations in $\mathrm{M}$.

This proposition implies that $\mathbf{A}$ is cofibrant if for every $a \in \mathrm{E}(\mathcal{D})$, and thus every diagram of the form (3.1), the corresponding diagram in $M$ has the form:

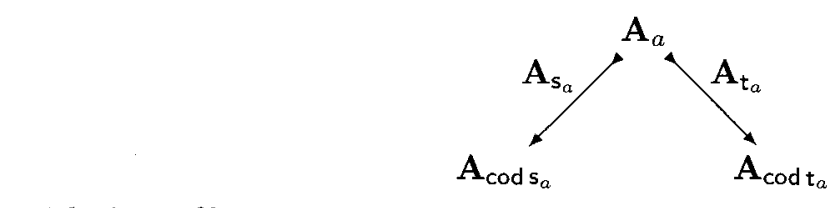

with $\mathbf{A}_{a}$ cofibrant. 
Proof. The initial object in $M^{\mathcal{D}}$ is given by $\Delta(\emptyset)$, where $\emptyset$ is the initial object of $\mathrm{M}$. Consider the morphism $\vec{f}: \Delta(\emptyset) \dot{\rightarrow} \mathbf{A}$. Clearly, for $a \in \mathrm{E}(\mathcal{D})$,

$$
i_{a}(\vec{f})=\vec{f}_{a}: \emptyset \rightarrow \mathbf{A}_{a}
$$

is a cofibration since $\mathbf{A}_{a}$ is cofibrant. For $b \in \mathrm{V}(\mathcal{D})$, the pushout diagram defining $i_{b}(\vec{f})$ becomes:

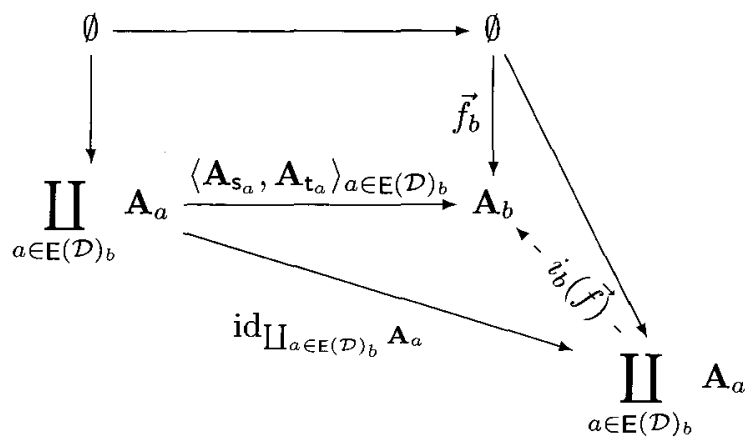

Therefore, for all $b \in \mathrm{V}(\mathcal{D}), i_{b}(\vec{f})=\left\langle\mathbf{A}_{\mathbf{s}_{a}}, \mathbf{A}_{\mathbf{t}_{a}}\right\rangle_{a \in \mathbf{E}(\mathcal{D})_{b}}$. To verify that this is a cofibration, we utilize Proposition 3.13 of [DS95]. That is, we show that $i_{b}(\vec{f})$ has the LLP with respect to acyclic fibrations. Consider a commuting diagram:

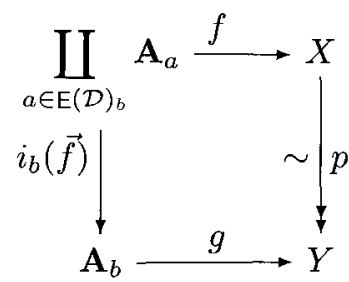

where $p$ is an acyclic fibration. For every $b \in \mathrm{E}(\mathcal{D})_{a}$ there is an associated diagram

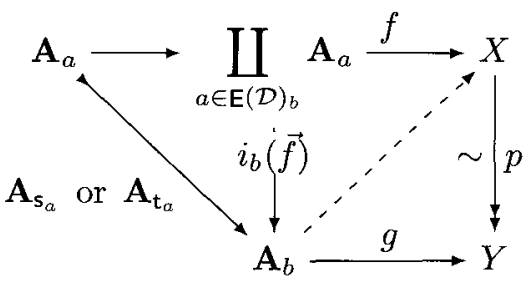

with the far right arrow either $\mathbf{A}_{\mathbf{s}_{a}}$ or $\mathbf{A}_{\mathbf{t}_{\alpha}}$. In both cases, the dashed arrow exists by the assumption that these morphisms are cofibrations. Since this holds for all $a \in \mathrm{E}(\mathcal{D})_{b}$, the dashed arrow therefore provides the desired lift for $i_{b}(\vec{f})$.

6.2. Computing homotopy pushouts. In light of Corollary 6.2 , an important aspect of computing homotopy colimits is being able to, given an object $\mathbf{A}$ of $\mathbf{M}^{\mathcal{D}}$, compute a cofibrant object $\mathbf{A}^{\prime}$ weakly equivalent to $\mathbf{A}$. For general Dcategories this is difficult, although a general method for doing so is presented in [Ame07]. In the case when

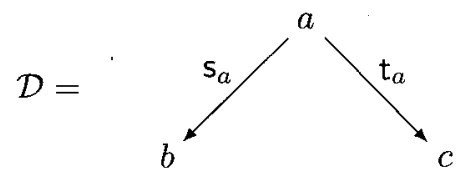


this is a simple matter. We will demonstrate this to give a sense of what the general procedure entails.

Let $\mathbf{A}$ be an object of $\mathrm{M}^{b \leftarrow a \rightarrow c}$. If $Q \mathbf{A}_{a}$ is a cofibrant replacement of $\mathbf{A}_{a}$, then we have a commuting diagram:

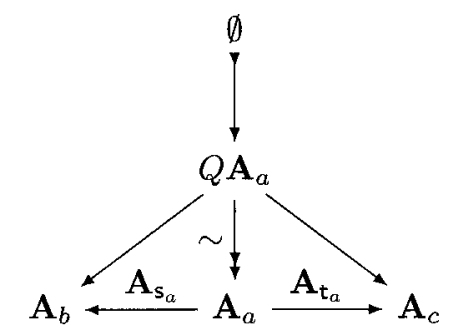

Factoring the left and right diagonal morphisms by MC5(1) for M yields a commuting diagram:

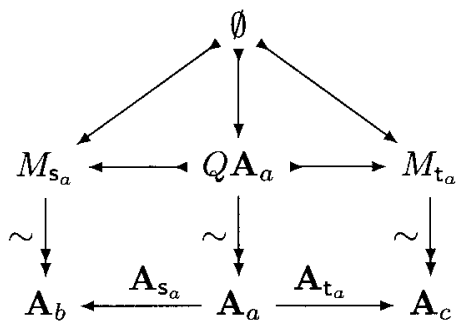

Let

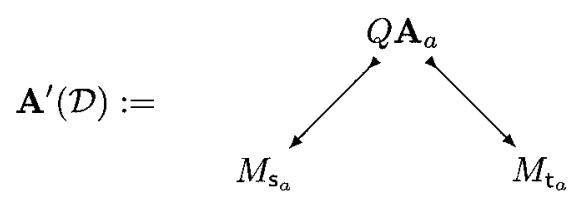

which is a cofibrant object in $\mathrm{M}^{b \leftarrow a \rightarrow c}$ weakly equivalent to $\mathbf{A}$. Therefore, the homotopy colimit of $\mathbf{A}$, termed the homotopy pushout, is given by:

$$
\operatorname{hocolim}(\mathbf{A}) \cong M_{\mathbf{s}_{a}} \mathrm{II}_{Q \mathbf{A}_{a}} M_{\mathrm{t}_{a}} \text {. }
$$

These concepts can be further illustrated in the context of topological spaces.

6.3. Homotopy pushouts in Top. To illustrate how one applies the previous ideas in a concrete setting, we will explicitly construct homotopy pushouts for topological spaces. It will be seen that the resulting topological space is in fact the space that one would expect.

Recall that for any map $f: X \rightarrow Y$ between topological spaces, there exists a factorization of this map:

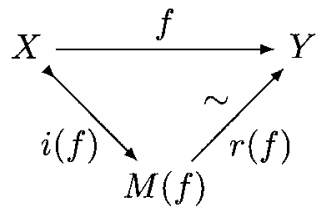

where

$$
M(f)=\frac{Y \amalg(X \times I)}{f(a) \sim(a, 0), a \in X}
$$


is the mapping cylinder, $i(f): X \rightarrow M(f)$, i.e., the inclusion sending $X$ to $X \times$ $\{1\} \subset M(f)$, is a cofibration and $r(f): M(f) \rightarrow Y$ is a homotopy equivalence (see [Pic92]).

Now consider a hybrid topological space $\mathbf{X}: \mathcal{D} \rightarrow$ Top with $\mathcal{D}$ as in (6.1), i.e., an object in Top ${ }^{b \leftarrow a \rightarrow c}$. The goal is to compute the homotopy pushout of this hybrid object. Using the mapping cylinder construction, we obtain a diagram of topological spaces:

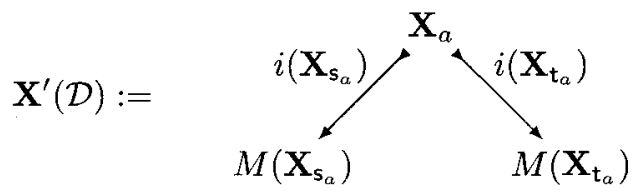

where $\mathbf{X}_{a}$ is cofibrant (since every topological space is cofibrant) with $i\left(\mathbf{X}_{\mathbf{s}_{a}}\right)$ and $i\left(\mathbf{X}_{\mathbf{t}_{a}}\right)$ cofibrations. Hence, $\mathbf{X}^{\prime}$ is a cofibrant object in Top ${ }^{b \leftarrow a \rightarrow c}$. Finally, the following diagram

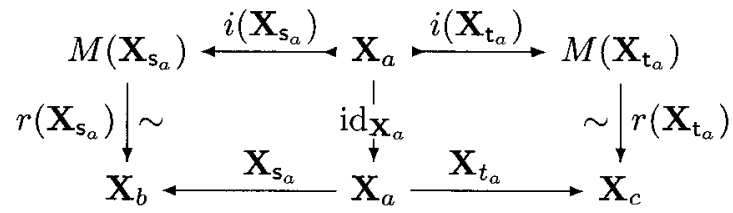

commutes, and so $\mathbf{X}^{\prime}$ is weakly equivalent to $\mathbf{X}$. Summarizing, it follows that for every object $\mathbf{X}$ of Top ${ }^{b \leftarrow a \rightarrow c}$

$$
\begin{aligned}
\operatorname{hocolim}(\mathbf{X}) & \cong M\left(\mathbf{X}_{\mathrm{s}_{a}}\right) \amalg_{\mathbf{X}_{a}} M\left(\mathbf{X}_{\mathrm{t}_{a}}\right) \\
& \cong \frac{\mathbf{X}_{b} \amalg \mathbf{X}_{c} \amalg\left(\mathbf{X}_{a} \times I\right)}{\mathbf{X}_{\mathbf{s}_{a}}(x) \sim(x, 0), \quad \mathbf{X}_{\mathrm{t}_{a}}(x) \sim(x, 1), \quad x \in \mathbf{X}_{a}}
\end{aligned}
$$

where the second isomorphism (in Ho(Top), hence homotopy equivalence) essentially is given by contracting $[0,2]$ to $[0,1]$. Therefore, we have recovered the standard homotopy pushout through the general framework of model category theory; that is, we have recovered the homotopy pushout "axiomatically."

More generally, utilizing the explicit formula for homotopy colimits in Top given in [Vog73], in [AS05] it was shown (see [Ame06b] for a proof) that the homotopy colimit of a general hybrid topological space $\mathbf{X}: \mathcal{D} \rightarrow$ Top is given by:

$$
\operatorname{hocolim}(\mathbf{X})=\frac{\left(\coprod_{b \in \mathrm{V}(\mathcal{D})} \mathbf{X}_{b}\right) \amalg\left(\coprod_{a \in \mathrm{E}(\mathcal{D})}\left(\mathbf{X}_{a} \times I\right)\right)}{(x, 0) \sim \mathbf{X}_{\mathbf{s}_{a}}(x), \quad(x, 1) \sim \mathbf{X}_{\mathbf{t}_{a}}(x), \quad x \in \mathbf{X}_{a}, \quad a \in \mathrm{E}(\mathcal{D})} .
$$

It is important to note that this formula was not derived axiomatically through the framework of model category theory; therefore, results like Corollary 6.2 do not follow automatically. The goal of [Ame07] is to derive explicit formulas for the homotopy colimit of hybrid objects axiomatically and for general model categories.

\section{Conclusion}

In this paper, we demonstrated that there exists a cofibrantly homotopy meaningful model structure on $M^{\mathcal{D}}$ for every model category $M$ and D-category $\mathcal{D}$. The homotopy theory on $\mathrm{M}^{\mathcal{D}}$ and the homotopy theory on $\mathrm{M}$ are thus related through homotopy colimits. The author believes that this result presents the first steps toward establishing a homotopy theory for hybrid systems. 
Acknowledgements. The author is indebted to Mariusz Wodzicki for the many insightful discussions on model categories and their role in hybrid systems, and to Shankar Sastry for his support.

\section{References}

[Ame06a] A. D. Ames, A categorical theory of hybrid systems, Ph.D. thesis, University of California, Berkeley, 2006.

[Ame06b] _ Hybrid model structures, Master's thesis, University of California, Berkeley, 2006.

[Ame07] - Hybrid model structures and homotopy colimits, In preparation, 2007.

[AS05] A. D. Ames and S. Sastry, A homology theory for hybrid systems: Hybrid homology, Hybrid Systems: Computation and Control (M. Morari and L. Thiele, eds.), Lecture Notes in Computer Science, vol. 3414, Springer-Verlag, 2005, pp. 86-102.

[AS06] _ Hybrid Routhian reduction of hybrid Lagrangians and Lagrangian hybrid systems, 25th American Control Conference (Minneapolis, MN), 2006.

[ATS06] A. D. Ames, P. Tabuada, and S. Sastry, On the stability of Zeno equilibria, Hybrid Systems: Computation and Control (J. Hespanha and A. Tiwari, eds.), Lecture Notes in Computer Science, vol. 3927, Springer-Verlag, 2006, pp. 34-48.

[Bro99] B. Brogliato, Nonsmooth mechanics, Springer-Verlag, 1999.

[CS02] W. Chachólski and J. Scherer, Homotopy theory of diagrams, vol. 155, Memoirs of the American Mathematical Society, no. 736, American Mathematica Society, 2002.

[DHKS04] W. G. Dwyer, P. S. Hirschhor, D. M. Kan, and J. H. Smith, Homotopy limit functors on model cateogries and homotopical categories, Mathematical Surveys and Monographs, vol. 113, American Mathematica Society, 2004.

[DS95] W. G. Dwyer and J. Spanlinski, Homotopy theories and model categories, pp. 73-126, Elsevier Science, 1995.

[Hat02] A. Hatcher, Algebraic topology, Cambridge University Press, 2002.

[Hov99] M. Hovey, Model categories, Mathematical Surveys and Monographs, vol. 63, American Mathematica Society, 1999.

[Lan98] S. Mac Lane, Categories for the working mathematician, second ed., Graduate Texts in Mathematics, vol. 5, Springer, 1998.

[MLS93] R. M. Murry, Z. Li, and S. Sastry, A mathematical introduction to robotic manipulation, CRC Press, 1993.

[Pic92] R. A. Piccinini, Lectures on homotopy theory, North-Holland mathematics studies, vol. 171, Elsevier Science, 1992.

[Qui67] D. Quillen, Homotopical Algebra (A. Dold and B. Eckmann, eds.), Lecture Notes in Mathematics, vol. 43, Springer-Verlag, 1967.

[SJSL00] S. Simic, K. H. Johansson, S. Sastry, and J. Lygeros, Towards a geometric theory of hybrid systems, HSCC (B. Krogh and N. Lynch, eds.), LNCS, vol. 1790, Springer Verlag, 2000, pp. 421-436.

[Str72] A. Strøm, The homotopy category is a homotopy category, Archiv der Mathematik 23 (1972), 435-441.

[Vog73] R. M. Vogt, Homotopy limits and colimits, Mathematische Zeitschrift 134 (1973), $11-52$.

Control and Dynamical Systems, California Institute of Technology, Pasadena. CA 91125

E-mail address: ames@cds.caltech.edu 